\title{
An Artificial Emotional Agent-Based Architecture for Games Simulation
}

\author{
Rainier Sales, Esteban Clua, Daniel de Oliveira, and Aline Paes \\ Universidade Federal Fluminense, Brazil \\ \{rsales, esteban, danielcmo, alinepaes\}@ic.uff.br
}

\begin{abstract}
Emotions are fundamental in in any person's life, and would be no different in games. While games are based at the gameplay for achieving the necessary fun factor, emotive elements are becoming common and in some cases even necessary. In some computing entertainment based systems, including and bringing emotional aspects from the real world may aspects of reality, turning the virtual characters more believable. This poster proposes a novel architecture for supporting Non-player character (NPC) behavior modeling, enhancing emotive agents. Personality, mood and emotive features are determined to the agents, based on well-established models developed in psychology. We validate our system in simulation games.
\end{abstract}

Keywords: Emotional Agents, Digital Games, Artificial Intelligence, NPC.

\section{Introduction}

Digital games are often considered closed systems, in the sense that there is no direct communication with the outside world events. Usually, they are only updated by the developers, either to improve playability or to fix bugs. However, the game may be-come more attractive to the player if real world events capable to interfere in the game are captured. The objective of this research is the creation of affective agents, also called emotional agents, and its inclusion at the gaming environment. The use of such agents in NPC follows the idea of the convenience for interpreting external events and bringing them inside the game. This research contributes with a novel architecture that aims to model NPC emotions and feelings more similar to the real humans. To achieve that, NPC in the game are mapped to artificial emotion-based agents, built from personality, emotion and mood models, previously developed in psychology science. In this sense, emotion is recognized as a central element of human behavior, and we intend to simulate within the game real situations regarding the game's domain.

\section{Representation and Modeling of Psychological Aspects with Emotional Agents}

The representation of real psychological aspects of characters in games is an essential part of this research. In this section we present the models of psychology 
that we will adopt in our architecture. The characters will be built based on four models that can communicate in an acceptable manner, namely: Personality model, emotion model, mood model and response model. Games traditionally implements agent events as game triggers that executes some kind of specific action. Our proposal intends to capture and compute events or inputs from the external world, in order to increase human aspects, such as personality, mood, emotion and response.

\subsection{Models: Personality, Emotion, Mood and Response}

The psychology model chosen for modeling the personality of the artificial agent in the proposed architecture is known as the Big Five model, taken from [1]. This is the model commonly used in recent research to describe personality of individuals ac-cording to a number of defined features.

Emotion is recognized as a central feature of human behavior. Because of that, it has been extensively studied in various areas of knowledge such as Psychology, Neu-roscience, Philosophy and Artificial Intelligence. In [2] emotions in the theory of evaluation are defined as the evaluation process, where events are classified and their emotional intensity is determined by the goals, beliefs, attitudes and risk of the person or agent. In the search for a suitable model for this process, we have chosen for this research the model of Cognitive Ortony, Clore and Collins (OCC), due to its simplicity of implementation in logic programming.

Mood and emotion are different in terms of intensity and duration [3]: mood dif-fers from emotion in the sense that are considered more durable and result from small events or stimuli generated from emotions. Because of that, besides mapping the basic personality and emotions of the artificial agent, it is also necessary to consider features based on its mood. Traditionally, the mood is represented in a one-dimensional scale, where the extremes are "good" and "bad" [4]. In order to describe and assess mood states, we used the PAD model (Pleasure, Arousal and Dominance) [5].

In our model, the agent will respond to some event that is linked to the three states of mood reactions described in the PAD model [6]. Such an answer defines the final behavior of the agent and is obtained directly from the eight possible combinations of mood, which in turn are related to the personality and emotional model of the agent. We resort to logical reasoning to conclude that the agent responds to an event in the game. When modeling human behavior to an artificial agent, personali-ty/emotional/mood features are not usually only totally true or false. They rather admit intermediate values between true and false. The fuzzy logic enables the capturing of vague information, commonly present in the natural language, in order to convert the results arising from the PAD model to a numeric value. For this research we define the Fuzzy Logic as a tool capable of capturing vague information, generally described in a natural language, as the results obtained in the model of mood PAD, mapping them to a numeric value, capable to be computationally manipulated. 


\section{Model Simulation}

This topic aims to present a simple simulation to test the applicability of the use of agents in games and emotional changes possible. It is believed that the theory and practice based here can add much in the way the players understand and play current games.

With the goal of presenting results, we developed a simple simulation, initially, it is necessary to introduce values in the five basic guidelines personality parameters. Then, from the personality, the simulator computes in real time which is the base mood of the agent. With the baseline mood set, the simulator calculates the current emotion of the agent. An emotion will be assigned only when this is greater than the minimum intensity perception. A particular action will be relevant to the agent when it has an associated emotion. When an event is perceived by the agent, we have to change its current emotion parameters. In this way, the emotion value assigned to the agent is no longer derived from the personality and base mood, but it also takes into account the external events.

There are several games that can make use of the architecture proposed here, among these we can highlight PES $2013^{T M}$, NBA2K13 ${ }^{T M}$, UFC UNDISPUTED $3^{T M}$, NHL $13^{T M}$, where the events represented internally to the game are the same hap-pened in the real world. In order to show the applicability of the architecture present-ed in this poster, we introduce a simulation of the game FIFA $13^{T M}$, where we used the original data of the same. In the database of FIFA 13 game each player has a specific configuration based on the performance of the real world, which is going to be the way in which the performance intensity alters the actions of the player. This example has simple configurations for results, initially the system searches for the values in the database to display the information agent. By modifying the performance intensity of the agent from the entrance of an event, we are going to have a different value related to attribute penalties. To validate the results we used as basis the test presented below.

[Test 1]: "A Positive input event maximizes positive outputs response". In this case, a positive emotion maximizes the positive results. We considered the average results for each attribute in 100 tests for each of the 11 positive emotions, generating a total of 1100 tests to check this test. As results presented, all positive events produced positive results, changing to greater than half of the original value.

[Test 2]: "A Negative input event maximizes negative outputs response". In this case, a negative emotion maximizes the negative results, the average results for each attribute in 100 tests for each of the 11 negative emotions, generating a total of 1100 tests to check this test. As results presented, all negative results changed to less than half the original value.

[Test 3]: "Positive input events intensity generates different results of different intensity, respecting the test 1". To check this test will make use of the attribute performance minimum intensity equal to 0.5 . As the logic applied is the same in all emotions we chose emotion Admiration for example. The results obtained from 100 tests were conducted for each of the three intensities using emotion generating a total of 300 tests. As the results show, higher intensities 
tend to positive results and to the maximum value of default agent value. For lower values of intensity, but above the minimum value, intensity measure will have more distant performance of the maxi-mum value of the agent.

[Test 4]: "Entries intensity of negative events generates different results of different intensity, respecting the test 2". To check this test will make the use of attribute performance minimum intensity equal to 0.5. As the logic applied is the same in all emotions we chose emotion Anger for example. The results were obtained from 100 tests, conducted for each of the three intensities adopted in the emotion, yielding a total of 300 tests. As the results show, higher intensities have larger negative results, tending in the opposite direction to the default value. For lower values of intensity, but above the minimum value intensity, values indicate performance measures farther from the opposite value of default agent value.

\section{Conclusion}

Currently the globalized world has made information available quickly and in many cases free, the information of an agent as a real football player, basketball or cricket are quickly available in different media, especially the Internet. The approach of this poster believes that this fatality of the contemporary world is favorable towards add-ing veracity to the games. The approach adopted here goes further, seeking not only to insert changes of the external environment, but work internally as the consequences of external world internally modifying the game. We believe that the proposal presented here may make games more fun for the player, since it event outside the game will have a direct impact on the performance of NPC, increasing the degree of reality between the game and the world of the player. Is important to note that the topics discussed here are part of an ongoing research and so the approach was only to evaluate and test the proposed structure, this new mechanism with the insertion of personality mood and emotion can generate emotional NPC.

\section{References}

1. Gazzaniga, Heatherton, Michael, S., Todd, F.: Psychological Science: Mind, Brain and Behavior. Artmed, Porto Alegre (2005)

2. Strongman, Kenneth, T.: The Psychology of Emotion. John Wiley and Sons, Chichester (2003)

3. Vinayagamoorthy, Vinoba, Gillies, M., Steed, A., Tanguy, E., Pan, X., Loscos, C., Slater, E.M., et al.: Building expressions into virtual caracteres. In: Eurographics Conference State of the Art Reports, Vienna (2006)

4. Picard, Rosalind: Affective Computing. MIT Press, Boston (1997)

5. Mehrabian, Albert: Pleasure-arousal-dominance: A general framework for describing and measuring individual differences in temperament. Current Psychology (1995)

6. Leite, F.N.H.F.: Impact of emotional experiences in customer satisfaction: A study in medical offices. Universidade Federal de Fortaleza, Fortaleza - CE (2006) 